\title{
A study of 3D printed flexible supercapacitors onto silicone rubber substrates
}

\author{
Milad Areir $^{1}\left[\right.$ Yanmeng Xu $^{1} \cdot$ David Harrison $^{1} \cdot$ John Fyson $^{1}$
}

Received: 8 June 2017 / Accepted: 19 August 2017 / Published online: 6 September 2017

(c) The Author(s) 2017. This article is an open access publication

\begin{abstract}
The rapid development of flexible energy storage devices is crucial for various electronics industries. Highly flexible electrochemical double layer capacitors (EDLCs) can be manufactured by 3D printing technology. It was a great challenge to fabricate multiple material layers of the EDLC in one rapid and accurate deposition event. The fabricated structures were composed of twelve electrodes which could be configured in a number of different ways in one block module. This work aims to investigate the performance of the flexible EDLCs manufactured by 3D printing in a honeycomb pattern. The EDLC cells were fabricated using a slurry made from commercial activated carbon (AC) and a gel electrolyte deposited on a transparent silicone substrate. The flexible EDLCs structures can be used in flexible electronics with different patterns and sizes using 3D printer and can be applied to many applications such as wearable technology.
\end{abstract}

\section{Introduction}

Flexible energy storage devices such as EDLCs, also known as supercapacitors, show high power density during discharge. The EDLCs rely on the use of modern, lighter, and flexible electrode materials [1-27]. The main concept of the EDLC is described by the physicist Helmholtz as the EDLC relies on the accumulation of electrical charges on electrodes that are separated by electrolyte [1]. Several carbon and

Milad Areir

milad.areir@brunel.ac.uk

1 Department of Design, College of Engineering and Physical Sciences, Brunel University London, Uxbridge UB8 3PH, UK carbon-based materials have long been used as electrodes for EDLCs. Activated carbon (AC) is one of the most important commercial carbon materials being used as electrodes of EDLCs [2]. It is useful because of its high specific surface area, low-cost, an excellent synergy effect with additive materials and is easy to process. Recently, some attempts have been made to fabricate multiple material layers of the EDLC in one rapid and accurate deposition process [3]. Several fabrication processes have been used to fabricate flexible EDLCs and micro supercapacitors and reported in the literature including photolithography and an electrochemical deposition, a micro extrusion, coating, roll-to-roll printing, etc [4-20]. Compared to the method described here, other $3 \mathrm{D}$ printing methods are usually costly or not able to process all the materials necessary for making EDLCs, such as 3D selective laser melting machine [21]. 3D printing based on paste extrusion has been adopted for this study because it allows EDLCs to be fabricated in one single process including package sealing with various types of materials. In addition, EDLCs have been studied and manufactured by 3D direct-ink writing process, but the viscosity of the AC and silicone materials makes them unsuitable for this approach [22]. An important requirement of an EDLC nowadays is its ability to be highly flexible to meet the requirement for numerous applications. It is clear that some of these processes add little to the capacitance behaviour. Some need pre-treatment, e.g. the spray coating technique has a number of drawbacks like unevenness and a wide spread due to a high treatment temperature. The cost of fabrication is high and time-consuming when using the photolithography process. Limited electrode thicknesses and substrate are provided with the DVD burner process. Hence, these processes may not suit flexible EDLCs with a variety of material substrates, AC electrodes and gel electrolyte patterns and thicknesses. However, one of the major challenges of component 
utilisation in EDLCs is flexibility and electrochemical performance. The challenge is to develop a simple, rapid, and low cost process that does not require additional processing while fabricating highly flexible EDLCs. A method should achieve better accuracy and uniform coating, multi paradigm structures and be of high flexibility.

The additive layer manufacturing process has the ability to bring different geometries within samples, by creating different patterns and using many materials printed to make every sample [28]. The current improvement in multi material printing has been achieved by using open source 3D paste deposition printing. Material compatibility is an important consideration for successful printing and electrochemical performance of EDLCs. The three major layer types in EDLCs contain a small amount of binder, which has to be optimum in surface tension, binding strength and viscosity. Due to the requirement for homogenous rheology and flexibility of the binding material, the thermoplastic binder PVDF-HFP has been used in AC electrodes and current collectors. To avoid using organic electrolyte the PVDF-HFP was not used in the gel electrolyte [29, 30]. In this work, we provide a detailed description of the manufacture of highly flexible EDLCs using a 3D printing method, transferring honeycomb-patterned layers for EDLCs components that have been fabricated. A honeycomb pattern was used due to less material and less time being consumed than with other patterns. The electrochemical testing of six and twelve AC electrodes of $2 \mathrm{~mm}$ thickness using $2.4 \mathrm{M}$ concentration of $\mathrm{H}_{3} \mathrm{PO}_{4}$ has been investigated.

\section{Experimental}

\subsection{Materials}

Zinc metal powder (Zn, MW 65.38) was purchased from SciChem ${ }^{\circledR}$. Silver powder was purchased from Gwent Group $^{\circledR}$. Silver conductive paint with a volume resistivity of $0.001 \Omega \mathrm{cm}$ was purchased from $\mathrm{RS}^{\circledR}$ Components Ltd. Dow Corning ${ }^{\circledR}$ acetoxy silicone was supplied by Screwfix ${ }^{\circledR}$. All other materials including the AC powder (AR grade, MW $12.01 \mathrm{~g} \mathrm{~mol}^{-1}$ ), sodium carboxymethyl cellulose (CMC, MW 250,000), polyvinylidene fluoride-co-hexafluoropropylene (PVDF-HFP, MW 400,000 130,000), polyvinyl alcohol (PVA, MW 146,000 186,000, 99+\% hydrolysed), 1-methyl2-pyrrolidinone (NMP, $1.028 \mathrm{~g} \mathrm{ml}^{-1}$ ) and phosphoric acid $\left(\mathrm{H}_{3} \mathrm{PO}_{4}, 2.4 \mathrm{M}\right)$ were supplied by Sigma-Aldrich ${ }^{\circledR}$.

\subsection{Preparation of AC slurry}

A small amount of water and binder as a lubricant in 3D printing is imperative. PVDF-HFP cannot be easily introduced into the electrode manufacturing process, as it cannot be dissolved in water. Thus, PVDF-HFP powder was added to NMP, followed by subsequent heating to $40{ }^{\circ} \mathrm{C}$ for $1 \mathrm{~h}$ to give concentration of $20 \% \mathrm{wt} / \mathrm{vol} .4 \mathrm{~g}$ of $\mathrm{AC}$ powder was mixed with vigorous stirring without heating for $24 \mathrm{~h}$ to ensure homogeneity.

\subsection{Preparation of $\mathrm{PVA} / \mathrm{H}_{3} \mathrm{PO}_{4}$ gel electrolyte}

To prepare an aqueous solution of PVA, $2.4 \mathrm{~g}$ of PVA powder was mixed with $30 \mathrm{~mL}$ distilled water and was magnetic stirred at $50{ }^{\circ} \mathrm{C}$ for $1 \mathrm{~h}$ until fully dissolved. $1.5 \mathrm{~g}$ of CMC $5 \%$ was then mixed with the PVA solution followed by $7 \mathrm{M}$ $\mathrm{H}_{3} \mathrm{PO}_{4}$ with magnetic stirring overnight until it completely turns to a homogeneous gel.

\subsection{Current collector paste preparation}

To use the state-of-the-art binder in the conductive paste and to prevent oxidation, $1 \mathrm{~g}$ of PVDF-HFP pellets was dissolved in $5 \mathrm{~mL}$ of NMP at $40{ }^{\circ} \mathrm{C}$ for $1 \mathrm{~h}$. Afterward, $1 \mathrm{~g}$ of zinc metal powder was mixed with $3 \mathrm{~g}$ of silver powder and added to the solvent/polymer mixture and kept stirring overnight to obtain a homogenous paste.

\subsection{Fabrication process}

Figure 1 shows the practical 3D fabrication technique of the flexible EDLCs. An extrusion tool for material paste deposition with a single syringe driven by a linear stepper motor called Discov3ry ${ }^{\circledR}$ has been installed in the 3D printer Ultimaker ${ }^{\circledR}$ firmware, and manipulated by simplify $3 \mathrm{D}^{\circledR}$ software. The 3D printer setting for the heated platform was set to $10{ }^{\circ} \mathrm{C}$ and the extruder temperature was $20^{\circ} \mathrm{C}$. A $30 \mathrm{~mL}$ BD luer-lock syringe was used. A $3.5 \mathrm{~mm}$ inner diameter of PVC rubber tube with length of $38 \mathrm{~cm}$ was used. To reduce the amount of the three materials used in this work they were deposited in a $20 \%$ honeycomb infill pattern. A $0.6 \mathrm{~mm}$ diameter tapered nozzle and a $0.6 \mathrm{~mm}$ layer height were used. The feed-rate was fixed at 21,000 steps per mm at a printing speed of $67 \mathrm{~mm} \mathrm{~s}^{-1}$. To avoid trapping any air bubbles, the silicone was filled into the syringe without any purification using an applicator gun and other pastes by femaleto-female luer-lock coupling. The retraction function in the software was disabled to avoid hardware mechanical issues. A non-stick Teflon sheet was used on the printing platform.

\subsection{Fabrication of flexible EDLCs devices}

A computer aided design (CAD) was created with Solid Works ${ }^{\circledR}$ and converted to 3D printer instructions in a layer by layer using simplify3 $\mathrm{D}^{\circledR}$ software. Each layer was fabricated in sequence by 3D paste extrusion as shown in Fig. 1a. 


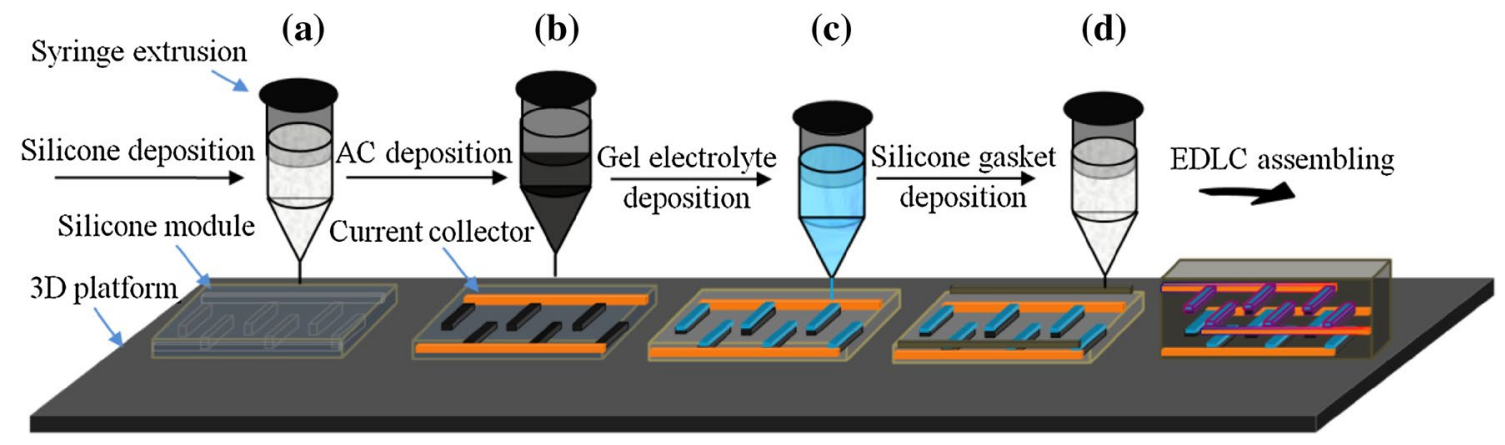

Fig. 1 Schematic illustration of the 3D fabrication procedures of flexible EDLCs

A silicone layer thickness of $0.6 \mathrm{~mm}$ was used throughout. Two layers of current collector applied with a brush, which served as current collectors. Electrode layer thicknesses of $0.6 \mathrm{~mm}$ were used throughout with dimension $38 \mathrm{~mm}$ length $\times 2 \mathrm{~mm}$ width Fig. 1b. The gel electrolyte dimensions were similar to the electrodes and this gel electrolyte layer was used as the separator. As shown in Fig. 1c the same 3D parameters for the $\mathrm{PVA} / \mathrm{H}_{3} \mathrm{PO}_{4}$ gel electrolyte layer were used for deposition to reach weight for the six electrodes of $1.3 \mathrm{~g}$. It has been demonstrated that various structures can be manufactured using silicone based 3D paste extrusion. The rheology of the paste materials needed to be controlled to allow deposition maintaining the filament shape. Pastes that have solvents such as NMP or acetone need to be printed at a low temperature to avoid evaporation. To minimize gel electrolyte leakage during the sandwiching of the two halves a $1 \mathrm{~mm}$ silicone layer was deposited around the edge Fig. 1d. The EDLC cells were printed without any metal current collector and kept in a vacuum desiccator for an hour to allow the infiltration into the carbon of the $\mathrm{PVA} / \mathrm{H}_{3} \mathrm{PO}_{4}$ electrolyte.

\subsection{Electrochemical measurements}

All measurements were carried out with an electrochemical workstation VersaSTAT 3.0 (Princeton Applied Research). A $2.4 \mathrm{M} \mathrm{PVA} / \mathrm{H}_{3} \mathrm{PO}_{4}$ was used as the gel electrolyte under ambient conditions. Cyclic voltammograms (CV) for the flexible EDLCs cell were recorded at different scan rates of 5, 10, 20, 50 and $200 \mathrm{mV} \mathrm{s}^{-1}$ between a potential voltage 0 and $0.8 \mathrm{~V}$. According to the equivalent circuit as shown in Fig. 2, the measured first capacitance $(\mathrm{Cl})$ and second capacitance $(\mathrm{C} 2)$ are given by Eq. (1). The twelve-electrode capacitance $(C 3)$ can be calculated from the Eq. (2).

$$
\begin{aligned}
& C 1=C 2=\frac{\left(\mathrm{Q}_{\text {total }} / 6\right)}{\Delta \mathrm{v}} \\
& C 3=\frac{\left(\mathrm{Q}_{\text {total }} / 12\right)}{\Delta \mathrm{v}}
\end{aligned}
$$

where $C$ is the capacitance in farads $(\mathrm{F}), \mathrm{Q}_{\text {total }}$ is the supercapacitor charge in coulombs $(\mathrm{C})$ and $\Delta \mathrm{v}$ is the voltage range in volts $(\mathrm{V})$. Galvanostatic charge/discharge (GCD) curves were measured at constant current densities of 11.53 and $5.76 \mathrm{~mA} \mathrm{~g}^{-1}$ for six and twelve electrodes, respectively. The determination of the GCD capacitance used by following equation

$C=\frac{\mathrm{i} \Delta \mathrm{t}}{\Delta \mathrm{v}}$

where $\mathrm{i}$ is the discharge current in amperes (A), $\Delta \mathrm{t}$ is the discharging time (s) and $\Delta \mathrm{v}$ is the voltage of the discharge $(\mathrm{V})$. The specific capacitance $\left(\mathrm{C}_{\mathrm{s}}\right)$ of the supercapacitor device can be calculated using

$\mathrm{C}_{\mathrm{s}}=\frac{\mathrm{i} \Delta \mathrm{t}}{\mathrm{m} \Delta \mathrm{v}}$

where $\mathrm{i}$ is the current in amperes (A), $\Delta \mathrm{t}$ is the discharging time (s), $\mathrm{m}$ is the total mass of the active materials of six and twelve electrodes $(\mathrm{g})$ and $\Delta \mathrm{v}$ is the voltage of the discharge with exception of iR drop (V).

\section{Results and discussion}

In this work, we present three EDLCs produced by a novel manufacturing process in a single module part. The schematic illustration of series connection without a combination circuit involved in the structure of the highly flexible EDLC is shown in Fig. 2. Based on the working mechanism [9], the EDLCs consist of four main components that are a substrate, current collector, and AC slurry and gel electrolyte as a separator. The stability of the EDLCs performance and its flexibility is mainly based on two factors, the vapour pressure of the solvent and the strength properties of the binders. Adhesive, common, cheap, and environmentally safe binders were used in the EDLCs. However, they tended to block a large part of the conductive particles, the carbon's surface area, and the electrolytes. The adhesion between the current 
Fig. 2 Schematic showing the steps used to connect the circuits of flexible EDLC

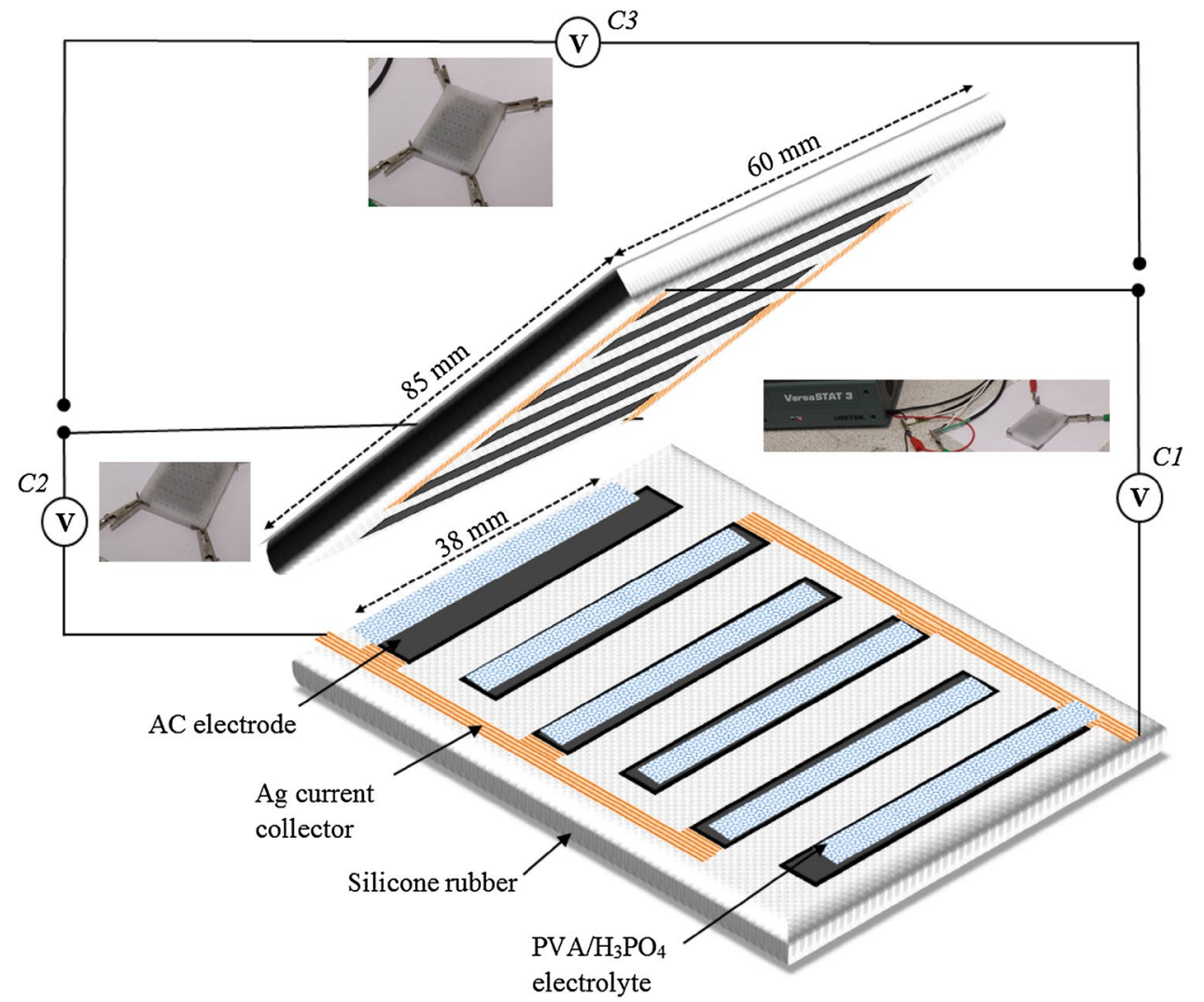

collector based on PVDF-HFP and the silicone substrate is weaker than that between other substrate elastic materials. The current collector based on PVDF-HFP showed a low resistance in comparison with current collector that had a CMC binder but the current collector paste based on CMC showed good flexibility in comparison with PVDF-HFP. The current collector based on PVDF-HFP was used as conducting layer in this work due to its high conductivity.

One of the fundamental challenges of 3D paste printing is that it is limited by syringe loading and the extrusion pressure required. The EDLC material was very challenging to deposit because it was prepared from powder and water leaving entrained air bubbles. This will cause breaks in the filament deposition leading to gaps and the structure to collapse. To optimise the structure, each layer must maintain its shape when printed. It is difficult to predict the shrinkage of EDLC materials components with low viscosity, as they spread after being deposited from the nozzle. Some acids and solvents are quick to evaporate during deposition, therefore; it is an important to select an appropriate acid and solvent. The rheological suspension properties of EDLC components are important in 3D printing processes and need to be controlled. The viscosity, surface tension, and AC particle content were adjusted to the requirements of the $0.6 \mathrm{~mm}$ tapered print nozzle. As shown in Fig. 3, the two layers with $0.6 \mathrm{~mm}$ of top shell

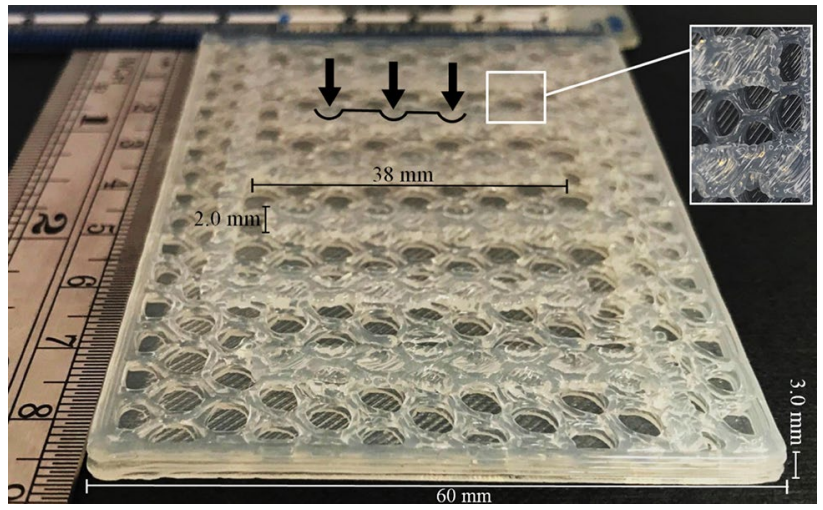

thickness in $20 \%$ honeycomb infill pattern lead to silicone layer shrinkage and distortion. To avoid an unsmooth skin surface on the honeycomb infill pattern it was necessary to increase the fill density above $20 \%$. It is very important to obtain excellent printed layers of silicone substrate and avoid formation of large gaps. This will reduce the defects within the current collector and AC layers and will lead to a uniform thickness and a stable electrochemical performance.
Fig. 3 Silicone honeycomb infill pattern exhibiting shrinkage layer noted by the lines 


\subsection{Cyclic voltammetry}

To determine the electrochemical properties of the AC printed onto silicone, it is highly important to take measurements at a sufficient scan rate by using $\mathrm{CV}$, the most widely used method for determining the capacitance. $\mathrm{CV}$ was performed in the potential range from 0 to $0.8 \mathrm{~V}$ to prevent the decomposition of water. The $\mathrm{CVs}$ were recorded in the aqueous $2.4 \mathrm{M} \mathrm{PVA} / \mathrm{H}_{3} \mathrm{PO}_{4}$ to determine $C 1, C 2$, and $C 3$. The mass of each electrode was $0.2 \mathrm{~g}$ and the total mass was $2.4 \mathrm{~g}$. The capacitance of $C 1$ and $C 2$ were calculated by Eq. (1) to be 264 , and $244.5 \mathrm{mF}$ at $5 \mathrm{mV} \mathrm{s}^{-1}$, respectively. The capacitance of $C 3$ was calculated by Eq. (2) to be $158.7 \mathrm{mF}$. The electrodes for $C l$ delivered capacitance of $151.49,83.98,37.79,20.15$ and $10.8 \mathrm{mF}$ when the scan rates rise to $10,20,50,100,200 \mathrm{mV} \mathrm{s}^{-1}$, respectively. At very slow charge rates, the ions can travel deeper inside the AC leading to a better surface coverage reaction. As shown in Fig. 4a, it can be clearly seen that the capacitance decreases because of the increase of the scan rate as the ions cannot get to the electrode surfaces quick enough. Other processes are also seen. A little reduction current was observed in $C 2$ at scan rate $200 \mathrm{mV} \mathrm{s}^{-1}$, which shows a faradaic (reaction) current. It is seen from Fig. $4 \mathrm{~b}$ that the three EDLCs display
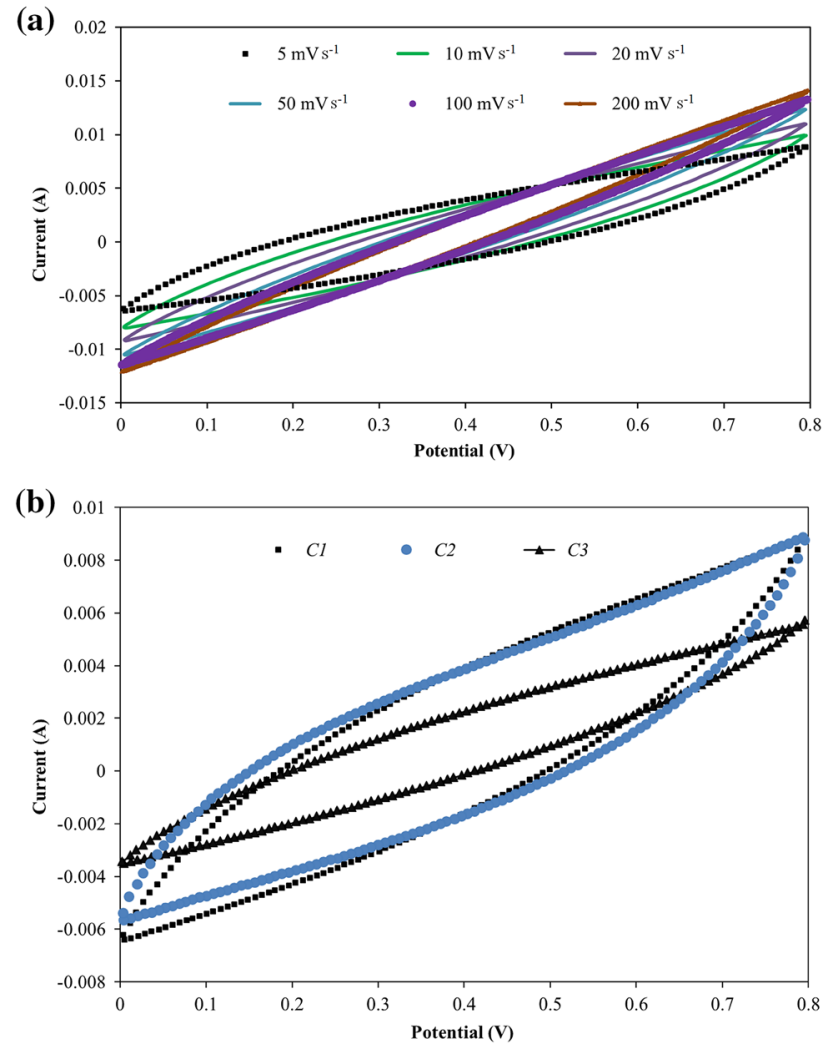

Fig. 4 a $\mathrm{CV}$ curves obtained from the $C 1$ at different scan rate; $\mathbf{b} \mathrm{CV}$ curves obtained from the three EDLCs at a scan rate of $5 \mathrm{mV} \mathrm{s}^{-1}$ a relatively good rectangular behaviour and the $C 1$ and $C 2$ showed the largest current at scan rate of $5 \mathrm{mV} \mathrm{s}^{-1}$ in comparison with $C 3$. However, the shrinkage of silicon surface in honeycomb pattern plays a role in changing dimensions and should be avoided because of their negative effect on EDLCs performance.

\subsection{Galvanostatic charge-discharge}

The GCD test was carried out for $C 1$ and $C 2$ at a current density of 11.5 and $5.7 \mathrm{~mA} \mathrm{~g}^{-1}$ for $C 3$ in the potential range 0 to $0.8 \mathrm{~V}$. Fig. 5 shows a typical fifth cycle charge-discharge curve for each EDLC. The capacitance for $\mathrm{Cl}$ calculated from the GCD curve using Eq. (3), is $11.2 \mathrm{mF}$, nearly half to that calculated from the $\mathrm{CV}$ at scan rate $100 \mathrm{mV} \mathrm{s}^{-1}$. There is little effect of current value and it can clearly show that the potential error $\left(\mathrm{V}_{\text {error }}\right)$ was extremely high in $C 3$ GCD curve. Uncompensated resistance might generate this error. The voltage drop (iR drop) at the beginning of each discharge curve was increased due to the high resistance and high voltage error. It was difficult to measure the iR drop and discharge time in $C 3$. Kampouris et al. [31] have considered the electrode to be polarized when the potential of an electrode is forced away from its open circuit. Similar negative slope trends of GCD curves for EDLC devices have been reported previously $[32,33]$. Because of uncompensated resistance, the impact of high $\mathrm{iR}$ drop on measurement is crucial. PVDF-HFP dissolved in NMP as binder is believed

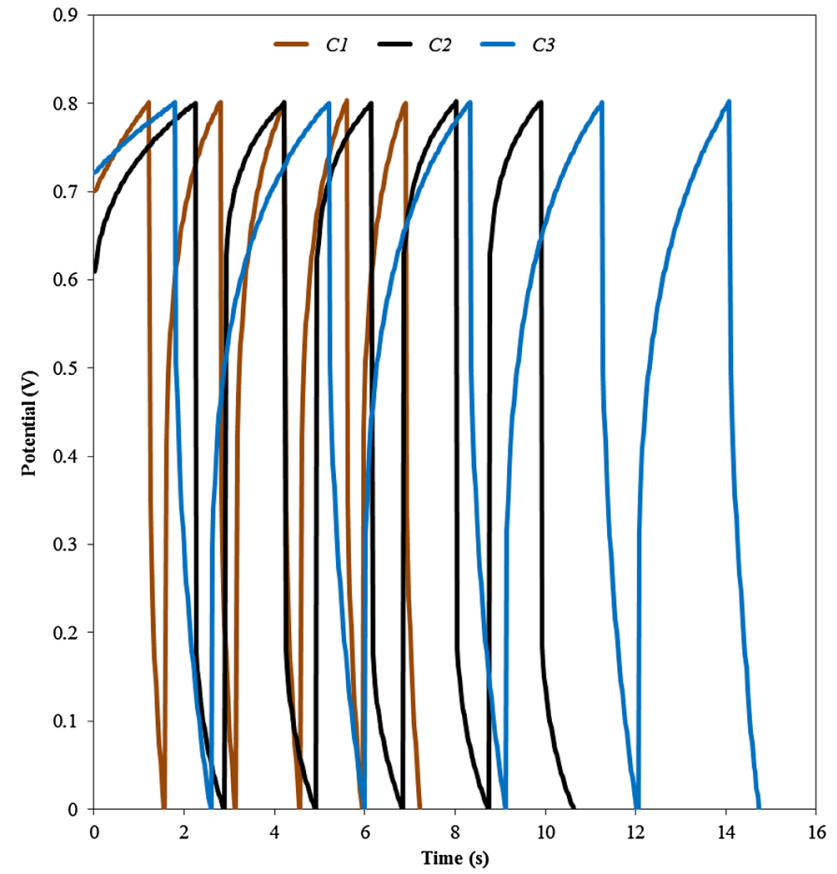

Fig. 5 GCD curves of the first five cycles of each EDLC at $0.8 \mathrm{~V}$ recorded at a charging current of $15 \mathrm{~mA}$ 
to be responsible for the scanning voltage going into the negative range during discharge process in GCD curves and away from the logical value of zero of aqueous electrolyte. Non-linear GCD curves are features of battery behaviour, which should not be used for measurement of capacitance values [34].

The values of specific capacitance were continually compared. The highest specific capacitance calculated by Eq. (4) was that reached at $7 \mathrm{mF} \mathrm{g}^{-1}$ for $C 1$. A minimum specific capacitance of $1 \mathrm{mF} \mathrm{g}^{-1}$ was observed for $C 3$ at a current density of $5.7 \mathrm{~mA} \mathrm{~g}^{-1}$. At a similar density for PVA/ $\mathrm{H}_{3} \mathrm{PO}_{4}$, the specific capacitance values of the flexible EDLC decreased when the charge/discharge current decreased. The energy density and power density are calculated from the GCD curves and equations (5) and (6).

$\mathrm{E}=\frac{1}{2} \mathrm{C}_{\mathrm{s}} \mathrm{v}^{2}$

$P=\frac{E}{\Delta t}$

where $\mathrm{E}$ is energy density, $\mathrm{C}_{\mathrm{s}}$ is the specific capacitance, $\mathrm{v}$ is the potential range, $\Delta \mathrm{t}$ is the discharging time, and $\mathrm{P}$ the power density. The highest energy density of the $C l$ was obtained to be $2.24 \mathrm{Ws} \mathrm{g}^{-1}$ with a corresponding power density of $3.18 \mathrm{~W} \mathrm{~g}^{-1}$. While the $C 3$ has lower energy density of $0.32 \mathrm{Ws} \mathrm{g}^{-1}$ with a corresponding power density of $1.6 \mathrm{~W} \mathrm{~g}^{-1}$.

\subsection{Electrochemical impedance spectroscopy (EIS)}

The equivalent series resistance (ESR) difference is explained in terms of the conductivity of $\mathrm{PVA} / \mathrm{H}_{3} \mathrm{PO}_{4}$. Other various factors may influence ESR such as electrode thickness and its attachment to the current collector, layer height, cell size, infill pattern and fill density. The ESR can be easily determined by obtaining a symmetrical GCD curve. An asymmetrical GCD curve is often difficult to measure due to the nonlinear slope. No noisy process was observed in any of the GCD curves. Thus, iR drop can be determined more reliably from the $\mathrm{iR}$ compensation technique ( $\mathrm{iR}_{\text {comp }}$ ) using the electrochemical workstation in auto-ranged experiment. The $\mathrm{iR}_{\text {comp }}$ were 14.9 and $18.86 \Omega$ for $C 1$ and $C 2$, respectively. $C 3$ displays a higher $\mathrm{iR}_{\text {comp }}$ was $41.37 \Omega$ because it might have been affected by remaining ionic resistance produced during the charging of $C 1$ and $C 2$. From the $\mathrm{iR}_{\text {comp }}$ values, we can calculate $\mathrm{V}_{\text {ir }}$ using the equation

$V_{\text {ir }}=\dot{i} R_{\text {comp }} \times \dot{i}_{\text {cell }}$

where $V_{i r}$ is the $i R$ drop at charge and discharge $(V)$ and $\mathrm{i}_{\text {cell }}$ is the current range in amperes (A). The values of $\mathrm{V}_{\text {ir }}$ are $0.22,0.28$, and $0.62 \mathrm{~V}$ for $C 1, C 2$, and $C 3$, respectively.
As shown in Fig. 6, ESR can be estimated for each EDLC from the intersection between the low frequency and more straight section of the Nyquist plot and the $\mathrm{X}$-axis [35]. The height of the 3D printed layer is $0.6 \mathrm{~mm}$ for AC electrodes and generally showed a weak adhesive force between electrodes and current collector resulting in poor cycle stability and high ESR. In addition, PVA/ $\mathrm{H}_{3} \mathrm{PO}_{4}$ aqueous gel electrolyte could not permeate quickly in comparison with the organic electrolyte. The AC based on PVDF-HFP binder showed a weight loss due to a loss of solvent leading to change in the layer thicknesses of electrode. The ion charge resistance is inversely proportional to the electrode area at $2 \mathrm{~mm}$ thickness. The expected semicircle cannot be seen in the higher frequency range due to the low contact resistance and the charge transfer resistance was low. It was unexpected that the bulk solution resistance of the $\mathrm{PVA} / \mathrm{H}_{3} \mathrm{PO}_{4}$ in $C 2$ was higher than $C l$ and $C 3$. The plot is horizontal at low frequency because of losing a quantity of electrons by Faradaic reactions or at $15 \mathrm{~mA}$ of charging current there has been an increase in the iR drop. The reduction of ESR and iR drop allows flexible EDLCs to achieve high power density. The main contribution of this work that a new approach to manufacture EDLC in a single process with various types of materials has been developed, which means EDLC can be manufactured to be the same or different form without the requirement for a mould pattern. AC, gel electrolyte, and silicone deposition by $3 \mathrm{D}$ printing process not only reduces the transferring pattern process and waste it also controls the layers and weight. The EDLCs were sealed as an integral part of this process without the need of additional laminating.

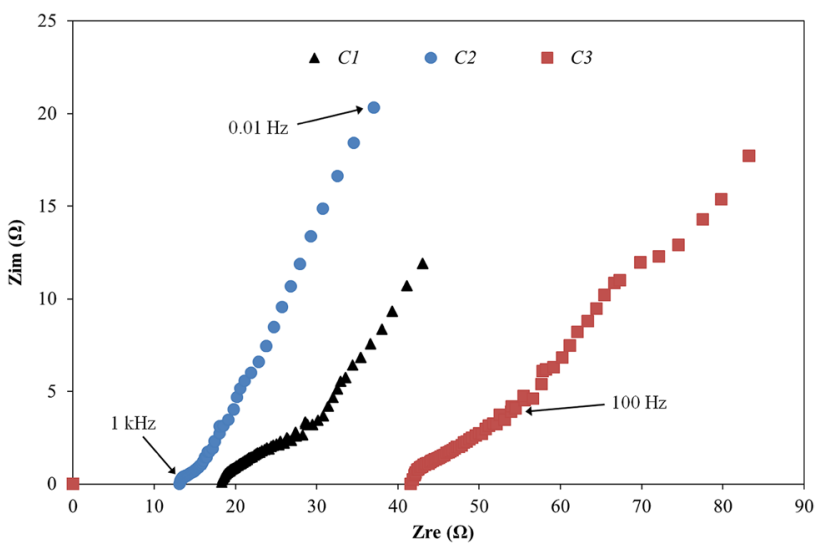

Fig. 6 Nyquist plot recorded at potential of $0 \mathrm{~V}$ for a frequency ranged from $100 \mathrm{kHz}$ to $0.01 \mathrm{~Hz}$ 


\section{Conclusion}

Flexible EDLCs with a silicone rubber-like substrate were fabricated successfully by 3D printing technology. It has been shown that $3 \mathrm{D}$ printing is a promising technique to make various patterns and accurate shaped structures using multiple materials that have different mechanical properties. Only one single extrusion device was responsible for building the structure described in this work. Three cells have been deposited in one module in a honeycomb pattern at a feed rate of 21,000 steps per mm. The reversing of the electrode polarity on discharge has been investigated and the electrochemical performances were tested at a potential of $0.8 \mathrm{~V}$ with a $2.4 \mathrm{M} \mathrm{PVA} / \mathrm{H}_{3} \mathrm{PO}_{4}$ gel electrolyte. The reduction of internal resistance has been shown to be dependent on the binder strength and thickness, pattern structure and the fill density of electrodes, gel electrolyte, and current collector. With further improvements of the AC slurry, gel electrolyte, and print parameters, the resolution of less than $0.6 \mathrm{~mm}$ should be achievable to maintain electrochemically stable flexible EDLCs. The optimized parameters can be applied to other devices and the manufacturing of 3D electronic structures for consumers. To satisfy the needs of electronic devices, the mechanical properties of lightweight and flexible energy storage devises fabricated by 3D printing will be explored in the future. The performance of combined circuits of the flexible EDLCs in parallel or in series will be examined in future work.

Acknowledgements This work was financially supported by the Ministry of Higher Education and Scientific Research of Libya.

Open Access This article is distributed under the terms of the Creative Commons Attribution 4.0 International License (http://creativecommons.org/licenses/by/4.0/), which permits unrestricted use, distribution, and reproduction in any medium, provided you give appropriate credit to the original author(s) and the source, provide a link to the Creative Commons license, and indicate if changes were made.

\section{References}

1. A.G. Pandolfo, A.F. Hollenkamp, Carbon properties and their role in supercapacitors. J. Power Sources 157, 11-27 (2006)

2. R. Zhang, Y. Xu, D. Harrison, J. Fyson, D. Southee, Experimental design to optimise electrical performance of strip supercapacitors. Int. J. Electrochem. Sci 11, 675-684 (2016)

3. C. Zhao, C. Wang, R. Gorkin, S. Beirne, K. Shu, G.G. Wallace, Three dimensional (3D) printed electrodes for interdigitated supercapacitors. Electrochem. Commun. 41, 20-23 (2014)

4. N. Kurra, M.K. Hota, H.N. Alshareef, Conducting polymer microsupercapacitors for flexible energy storage and Ac line-filtering. Nano Energy 13, 500-508 (2015)

5. J.H. Sung, S.J. Kim, S.H. Jeong, E.H. Kim, K.H. Lee, Flexible micro-supercapacitors. J. Power Sources 162, 1467-1470 (2006)
6. G. Sun, J. An, C.K. Chua, H. Pang, J. Zhang, P. Chen, Layer-bylayer printing of laminated graphene-based interdigitated microelectrodes for flexible planar micro-supercapacitors. Electrochem. Commun. 51, 33-36 (2015)

7. B. Gao, D. He, B. Yan, H. Suo, C. Zhao, Flexible carbon cloth based polypyrrole for an electrochemical supercapacitor. J. Mater. Sci. 26, 6373-6379 (2015)

8. Q. Zhou, X. Ye, Z. Wan, C. Jia, A three-dimensional flexible supercapacitor with enhanced performance based on lightweight, conductive graphene-cotton fabric electrode. J. Power Sources 296, 186-196 (2015)

9. R.R. Zhang, Y.M. Xu, D. Harrison, J. Fyson, F.L. Qiu, D. Southee, Flexible strip supercapacitors for future energy storage. Int. J. Autom. Comput. 12, 43-49 (2015)

10. N. Jiang, F. Huang, W. Xia, J. Wei, L. Zhou, Z. Huo, Q. Pang, Facile fabrication of rGO/CNT hybrid fibers for high-performance flexible supercapacitors. J. Mater. Sci. 28, 12147 (2017). doi:10.1007/s10854-017-7029-9

11. F. Qiu, D. Harrison, J. Fyson, D. Southee, Fabrication and characterisation of flexible coaxial thin thread supercapacitors. Smart Sci. 2, 107-115 (2014)

12. H. Durou, D. Pech, D. Colin, P. Simon, P.-L. Taberna, M. Brunet, Wafer-level fabrication process for fully encapsulated micro-supercapacitors with high specific energy. Microsyst. Technol. 18, 467-473 (2012)

13. B. Yao, J. Zhang, T. Kou, Y. Song, T. Liu, Y. Li, Paper-based electrodes for flexible energy storage devices. Adv. Sci. 4, 1700107 (2017). doi:10.1002/advs.201700107

14. J. Yeo, G. Kim, S. Hong, M.S. Kim, D. Kim, J. Lee, H.B. Lee, J. Kwon, Y.D. Suh, H.W. Kang, H.J. Sung, J.H. Choi, W.H. Hong, J.M. Ko, S.H. Lee, S.H. Choa, S.H. Ko, Flexible supercapacitor fabrication by room temperature rapid laser processing of rollto-roll printed metal nanoparticle ink for wearable electronics application. J. Power Sources 246, 562-568 (2014)

15. R.S. Ingole, B.J. Lokhande, Spray pyrolysed vanadium oxide thin films using different ingredients for redox supercapacitor. J. Mater. Sci. 27, 1363-1369 (2016)

16. M.F. El-Kady, R.B. Kaner, Scalable fabrication of high-power graphene micro-supercapacitors for flexible and on-chip energy storage. Nat. Commun. 4, 1475 (2013)

17. L. Yuan, B. Yao, B. Hu, K. Huo, W. Chen, J. Zhou, Polypyrrole-coated paper for flexible solid-state energy storage. Energy Environ. Sci. 6, 470-476 (2013)

18. H. Pang, Y. Zhang, W.Y. Lai, Z. Hu, W. Huang, Lamellar $\mathrm{K}_{2}$ $\mathrm{Co}_{3}\left(\mathrm{P}_{2} \mathrm{O}_{7}\right) 2 \cdot 2 \mathrm{H}_{2} \mathrm{O}$ nanocrystal whiskers: High-performance flexible all-solid-state asymmetric micro-supercapacitors via inkjet printing. Nano Energy 15, 303-312 (2015)

19. M. Areir, Y. Xu, R. Zhang, D. Harrison, J. Fyson, E. Pei, A study of 3D printed active carbon electrode for the manufacture of electric double-layer capacitors. J. Manuf. Process 25, 351-356 (2017)

20. B. Yao, L. Huang, J. Zhang, X. Gao, J. Wu, Y. Cheng, X. Xiao, B. Wang, Y. Li, J. Zhou, Flexible transparent molybdenum trioxide nanopaper for energy storage. Adv. Mater. 28, 6353-6358 (2016). doi:10.1002/adma.201600529

21. C. Zhu, T. Liu, F. Qian, W. Chen, S. Chandrasekaran, B. Yao, Y. Song, E.B. Duoss, J.D. Kuntz, C.M. Spadaccini, M.A. Worsley, Y. Li, 3D printed functional nanomaterials for electrochemical energy storage. Nano Today 1, 1-14 (2017). doi:10.1016/j. nantod.2017.06.007

22. C. Zhu, T. Liu, F. Qian, T.Y.J. Han, E.B. Duoss, J.D. Kuntz, C.M. Spadaccini, M.A. Worsley, Y. Li, Supercapacitors based on three-dimensional hierarchical graphene aerogels with periodic macropores. Nano Lett. 16, 3448 (2016). doi:10.1021/acs. nanolett.5b04965 
23. P. Wang, Y. Li, S. Li, X. Liao, S. Sun, Water-promoted zeolitic imidazolate framework- 67 transformation to Ni-Co layered double hydroxide hollow microsphere for supercapacitor electrode material. J. Mater. Sci. 13, 9221-9227 (2017). doi:10.1007/ s10854-017-6656-5

24. C.N. Chen, C.P. Chen, T.Y. Dong, T.C. Chang, M.C. Chen, H.T. Chen, I.G. Chen, Using nanoparticles as direct-injection printing ink to fabricate conductive silver features on a transparent flexible PET substrate at room temperature. Acta Mater. 60, 5914-5924 (2012)

25. T. Chen, L. Dai, Flexible supercapacitors based on carbon nanomaterials. J. Mater. Chem. A 2, 10756-10775 (2014)

26. Z. Yang, J. Deng, X. Chen, J. Ren, H. Peng, A highly stretchable, fiber-shaped supercapacitor, Angew. Chemie Int. Ed. 52, 13453-13457 (2013)

27. Z. Zhang, J. Deng, X. Li, Z. Yang, S. He, X. Chen, G. Guan, J. Ren, H. Peng, Superelastic supercapacitors with high performances during stretching. Adv. Mater. 27, 356-362 (2015)

28. W. Oropallo, L.A. Piegl, Ten challenges in $3 \mathrm{D}$ printing. Eng. Comput. 32, 135-148 (2016)

29. N. Böckenfeld, S.S. Jeong, M. Winter, S. Passerini, A. Balducci, Natural, cheap and environmentally friendly binder for supercapacitors. J. Power Sources 221, 14-20 (2013)
30. J.H. Shin, S.S. Jung, K.W. Kim, H.J. Ahn, Preparation and characterization of plasticized polymer electrolytes based on the PVdF-HFP copolymer for lithium/sulfur battery. J. Mater. Sci. 13, 727-733 (2002)

31. D.K. Kampouris, X. Ji, E.P. Randviir, C.E. Banks, A new approach for the improved interpretation of capacitance measurements for materials utilised in energy storage. RSC Adv. 5, 12782-12791 (2015)

32. Y. Zhang, B. Lin, J. Wang, J. Tian, Y. Sun, X. Zhang, H. Yang, All-solid-state asymmetric supercapacitors based on $\mathrm{ZnO}$ quantum dots/carbon/CNT and porous N-doped carbon/CNT electrodes derived from a single ZIF-8/CNT template. J. Mater. Chem. A 4, 10282-10293 (2016)

33. R. Ramachandran, M. Saranya, C. Santhosh, V. Velmurugan, B.P.C. Raghupathy, S.K. Jeong, A.N. Grace, Co9S8 nanoflakes on graphene (Co9S 8/G) nanocomposites for high performance supercapacitors. RSC Adv. 4, 21151-21162 (2014)

34. L. Guan, L. Yu, G.Z. Chen, Capacitive and non-capacitive faradaic charge storage. Electrochim. Acta 206, 464 (2016). doi:10.1016/j. electacta.2016.01.213

35. C. Lei, F. Markoulidis, Z. Ashitaka, C. Lekakou, Reduction of porous carbon/Al contact resistance for an electric double-layer capacitor (EDLC). Electrochim. Acta 92, 183-187 (2013) 